\title{
The Role of Higher-Level Cognitive Function in Gait: Executive Dysfunction Contributes to Fall Risk in Alzheimer's Disease
}

\author{
Pamela L. Sheridan ${ }^{a, b}$ Jeffrey M. Hausdorff ${ }^{b, c}$ \\ ${ }^{a}$ Behavioral Neurology Division, Department of Neurology, Beth Israel Deaconess Medical Center, and \\ ${ }^{b}$ Division on Aging, Harvard Medical School, Boston, Mass., USA; ${ }^{c}$ Movement Disorders Unit, \\ Tel-Aviv Sourasky Medical Center; Sackler School of Medicine, Tel-Aviv University, Tel-Aviv, Israel
}

\section{Key Words}

Gait · Cognition - Executive function · Attention •

Motor control $\cdot$ Alzheimer's disease

\begin{abstract}
Alzheimer's disease (AD) is generally understood as primarily affecting cognition while sparing motor function, at least until the later stages of the disease. Studies reported over the past 10 years, however, have documented a prevalence of falls in AD patients significantly higher than in age-matched normal elders; also persons with $A D$ have been observed to have different walking patterns with characteristics that increase gait instability. Recent work in cognitive neuroscience has begun to demonstrate the necessity of intact cognition, particularly executive function, for competent motor control. We put the pieces of this puzzle together and review the current state of knowledge about gait and cognition in general along with an exploration of the association between dementia, gait impairment and falls in AD. We also briefly examine the current treatment of gait instability in $A D$, mainly exercise, and propose a new approach targeting cognition. Copyright $\odot 2007$ S. Karger AG, Basel
\end{abstract}

\section{Introduction}

Alzheimer's disease (AD), a degenerative brain disease that primarily affects cognition, is generally understood as sparing motor function and gait early in the disease process. This idea has been sustained by a large body of research that has not demonstrated abnormalities in areas of the central nervous system primarily involved with motor control [1,2].

Recent work, however, is beginning to challenge this notion.

Walking is generally considered to be an automatic process involving little or no higher cognitive input $[3,4]$. Thus it is somewhat surprising that patients with $\mathrm{AD}$ have a markedly increased fall risk; the rate is almost three times more than that seen among nondemented elderly [5-9]. Recent reports, however, suggest that walking under usual circumstances may require attention and executive function [10-15], and changes in cognitive function may contribute to an increased fall risk.

As we explain in detail below, we suggest that, in contrast to the more traditional view, a growing body of literature supports the following ideas: (1) that walking may be viewed as a cognitive process requiring higher-level control, (2) that changes in cognitive function in AD contribute to gait disturbances and increase fall risk in $\mathrm{AD}$, and (3) that to reduce fall risk in $\mathrm{AD}$, a new cognitivebased approach is needed. More specifically, in this re-

Pamela Sheridan, MD, MMSc

Behavioral Neurology, Beth Israel Deaconess Medical Center

330 Brookline Avenue

Boston, MA 02215 (USA)

Tel. +1 617667 0483, Fax +1 617667 7981, E-Mail psherida@caregroup.harvard.edu

\begin{tabular}{ll}
\hline KARGER & ( 2007 S. Karger AG, Basel \\
1420-8008/07/0242-0125\$23.50/0 \\
$\begin{array}{l}\text { Fax +4161306 1234 } \\
\begin{array}{l}\text { E-Mail karger@karger.ch } \\
\text { www.karger.com }\end{array}\end{array}$ & $\begin{array}{l}\text { Accessible online at: } \\
\text { www.karger.com/dem }\end{array}$
\end{tabular}


view, we: (1) briefly summarize the evidence supporting the idea that walking is an automatic movement that does not require higher-level cognitive input; (2) review the recent work that challenges this hypothesis, and (3) provide evidence that suggests that cognitive impairment, in particular executive dysfunction, contributes to fall risk in $\mathrm{AD}$.

\section{Walking, Essentially an Automatic Movement, Actually Requires Cognitive Input}

A great deal of what we understand about walking has been obtained from studies in quadrupeds, particularly cats $[4,16]$. In terrestrial animals locomotion is described as movement across a flat surface while maintaining equilibrium [4]. In humans this involves rhythmic, alternating movement of the legs, stepping, while maintaining an upright posture [3, 4]. Stepping movements are programmed at the spinal cord level [3]. There is strong evidence for the existence of central pattern generators at this level in cats and other mammals, for example decerebrate cats are able to walk with an essentially normal pattern of stepping [4]. Infants at birth perform step-like movements if stimulated by peripheral stimuli [3]. Their inability to 'walk' is due only to immaturity of the neuronal network that maintains equilibrium and an upright posture.

To initiate stepping, incoming sensory information is integrated within multiple, ascending levels of the central nervous system including the spinal cord, brainstem, basal ganglia and thalamic areas $[3,4]$. Once learned, locomotion is initiated at the level of the supplementary motor area (SMA) of the frontal lobe and executed by the primary sensorimotor areas, basal ganglia, cerebellum and other brainstem and spinal cord motor centers automatically without input from higher cognition $[3,4,17]$. During routine walking, previously learned programs of movement are modified, if necessary, according to a continuous inflow of sensory information from all modalities of perception. This allows for stability of equilibrium, stance and stride.

Analysis of walking in humans has largely, until recently, been limited to clinical observations of the mechanics of gait and peripheral neurophysiologic techniques. Lately, experiments using new imaging and neurophysiologic techniques such as functional MRI (fMRI), single photon computed tomography (SPECT), positron emission tomography (PET), and transcranial magnetic stimulation (TMS) have re-examined various aspects of motor control and motor learning [18-26]. These studies are both confirming and refuting long-held ideas about movement automaticity.

In order to examine the cortical contributions to bipedal movements, 7 healthy adult subjects performing bicycling movements were scanned using oxygen-15 PET [18]. Compared to rest, active bicycling stimulated sites bilaterally in primary sensory and motor cortices along with the SMA and anterior cerebellum. When imagining the same movements, only the SMA was activated (bilaterally). An earlier study, using SPECT, found a similar pattern. Fourteen healthy adults were scanned after injection with technetium-99 (HM-PAO) during a 4-min regularly paced walking protocol and then again after resting supine [19]. The SMA, medial primary sensorimotor areas, striatum, cerebellar vermis and visual cortices were activated. Cognitive association areas involved with executive function, particularly attention were not activated in either study, confirming the long-held belief that locomotor functions are automatic or routine and thus do not require cognitive input.

On the other hand, a study using PET to compare brain activations during active locomotion with imagined walking showed different results. Six healthy adults were scanned using $\mathrm{H}_{2}{ }^{15} \mathrm{O}$ PET while imagining locomotor tasks from the first person perspective [20]. First they were scanned performing actual motor tasks including walking and walking with obstacles. Then they were scanned after lying supine and imagining the same tasks actually performed; electromyogram (EMG) activity was recorded while they were scanned during each experiment. A common pattern of activation was observed while standing: bilateral activation of the dorsal premotor cortices, precuneus; left dorso-lateral prefrontal cortex (DLPFC), inferior parietal lobule and the right posterior cingulate cortex. When imagery of locomotion occurred, additional activation was seen in the caudate along with increased blood flow in the left cuneus. Walking with obstacles added stimulation in the left SMA. EMGs demonstrated no evidence of activity during the imaginary motor experiments. This study demonstrates that activation of the cortex linked to higher cognitive control is involved in both automatic and intentional, goal-directed motor behavior.

Attention functions are involved with motor control when actions are intentional or willed. Actions are considered goal-directed behaviors involving movement $[17,27-29]$. Willed action is considered an intentional, goal-directed behavior that involves conscious awareness and focus of attention [27, 29]. This is to be differentiated from stimulus-driven actions or unconscious 
but voluntary movement such as, in the traditional view, walking. The difficulty lies in the fact that most actions in normal adults tend to be voluntary and goal directed. This does not imply that all parts of actions involve conscious awareness. For example: one makes a decision to make a cup of coffee. Walking towards the kitchen to start the process does not usually involve conscious awareness of the act of walking unless something unusual stimulates sensory perception such as pain in the leg or an obstacle in the walking path. So, we have both intention and automatic behavior. Once a movement becomes learned and routine, activation occurs in areas of the cortex predominantly involved with motor control: SMA, primary sensorimotor areas, basal ganglia and cerebellum. Intention involves the input of cognitive association areas such as the DLPFC, anterior cingulate gyrus and the posterior parietal, middle and superior temporal gyri, depending on the context of the movement [24-28, 30-32]. Performance of simple but bimanual tasks such as moving fingers on both hands simultaneously in a specified sequence activates brain areas involved in higher cognitive executive functions, both before and after learning the movement, suggesting that control of routine movement requires continued input from posterior sensory systems and integration of this information with previously learned motor programs [23, 25, 28, 29, 31].

A very recent study of the cat lends support to this hypothesis [33]. Using single cell recording techniques the authors showed activation of more than half of the cells in the prefrontal cortex during a 3-min walk on a treadmill. The same pattern of firing was found when the posterior parietal cortex or the dorsomedial thalamus was stimulated independently while the cats rested. They concluded that the prefrontal cortex is actively involved in control of locomotion, in a network that involves the posterior parietal cortex along with the dorsomedial thalamus.

The role of the hippocampus in motor control is evolving. Recent preclinical work suggests that the hippocampus and to a lesser extent, parahippocampal regions are essential for spatial encoding and working memory, particularly involving locomotion (perceptual-motor integration) [34-38]. The capacity to respond to new, unexpected stimuli is considered a basic mammalian behavior. A network function, it involves brain regions involved in memory storage, predominantly bilateral posterior cortical regions and the right hippocampus [34, 35, 38]. A constant, rhythmic, slow (4-12 Hz, theta) oscillation of electrical activity in the hippocanpus, discovered on elec- trophysiologic recordings in both animals and humans, has been linked to movement, particularly locomotion [34-38]. Thought to play a role in encoding and retrieval of movement-related sensory information under all conditions, recent studies in rodents have demonstrated changes in amplitude and frequency relate to specific situations, in particular situations of novel, active movement [36, 37]. In humans, hippocampal networks are particularly involved in the detection of stimulus novelty with evidence of 'on-line' monitoring of sensory input along with comparison to previously stored info [34]. A recent MRI examination of working memory in humans determined that the hippocampus has two distinct functions: long-term memory storage and working memory maintenance [38].

So, in summary, it appears as if the network of conscious, intentional motor behavior is likely separate from the system for automatic movement; however, at all parts of the scheme, cognitive association areas are involved. DLPFC encodes the goal or plan for movement; the SMA then decides the sequence of movements after input from the posterior parietal association areas and superior temporal gyri (perceptual integration). The hippocampus and parahippocampal regions contribute to spatial planning and working memory as it relates to incoming sensory information, particularly novel stimuli. The plan is then transferred to the primary motor cortex with simultaneous processing subcortically in the basal ganglia (longterm storage of motor programs) and cerebellum (control of timing) with subsequent transfer to the spinal cord interneurons, thought to be responsible for control of activation of muscle firing patterns, and finally to the motor neurons in the anterior cord. There is constant feedback and communication at all levels with the sensory system, particularly in regards to spatial information.

The anatomic network of motor control is thus interlinked with the network of higher-level cognitive function, in particular executive function, and includes the DLPFC, cingulate gyrus, SMA, parietal association areas, superior temporal gyrus as well as the basal ganglia and medial temporal lobes (hippocampus and parahippocampal areas) (fig. 1).

\section{Executive Functions, Necessary for Motor Behavior, Are Impaired in AD}

Executive functions refer to a variety of higher cognitive processes that modulate and use information from the posterior cortical sensory systems to produce behav- 
Fig. 1. This is a schematic of the network of intentional and automatic motor behavior. The conscious pathway is represented by continuous, bold arrows; the unconscious by broken arrows. The posterior cortex is involved in sensory integration and memory. The frontal cortex primarily plans, sequences and organizes movement and action. The basal ganglia are involved with long-term storage; the cerebellum controls timing. $\mathrm{PFC}=$ Prefrontal cortex; DLPFC $=$ dorso-lateral prefrontal cortex; FC = frontal cortex; SMA = supplementary motor cortex; $\mathrm{MC}=$ motor cortex; $\mathrm{PMC}=$ premotor cortex; $\mathrm{PC}=$ parietal cortex; $\mathrm{TL}=$ temporal lobe; $\mathrm{STG}=$ superior temporal gyrus; $\mathrm{H}=$ hippocampus; $\mathrm{PH}=$ parahippocampal regions; $\mathrm{OC}=$ occipital cortex; $\mathrm{BG}=$ basal ganglia; $\mathrm{CB}=$ cerebellum; $\mathrm{SC}=$ spinal cord; $\mathrm{AHC}=$ anterior horn motor neurons.

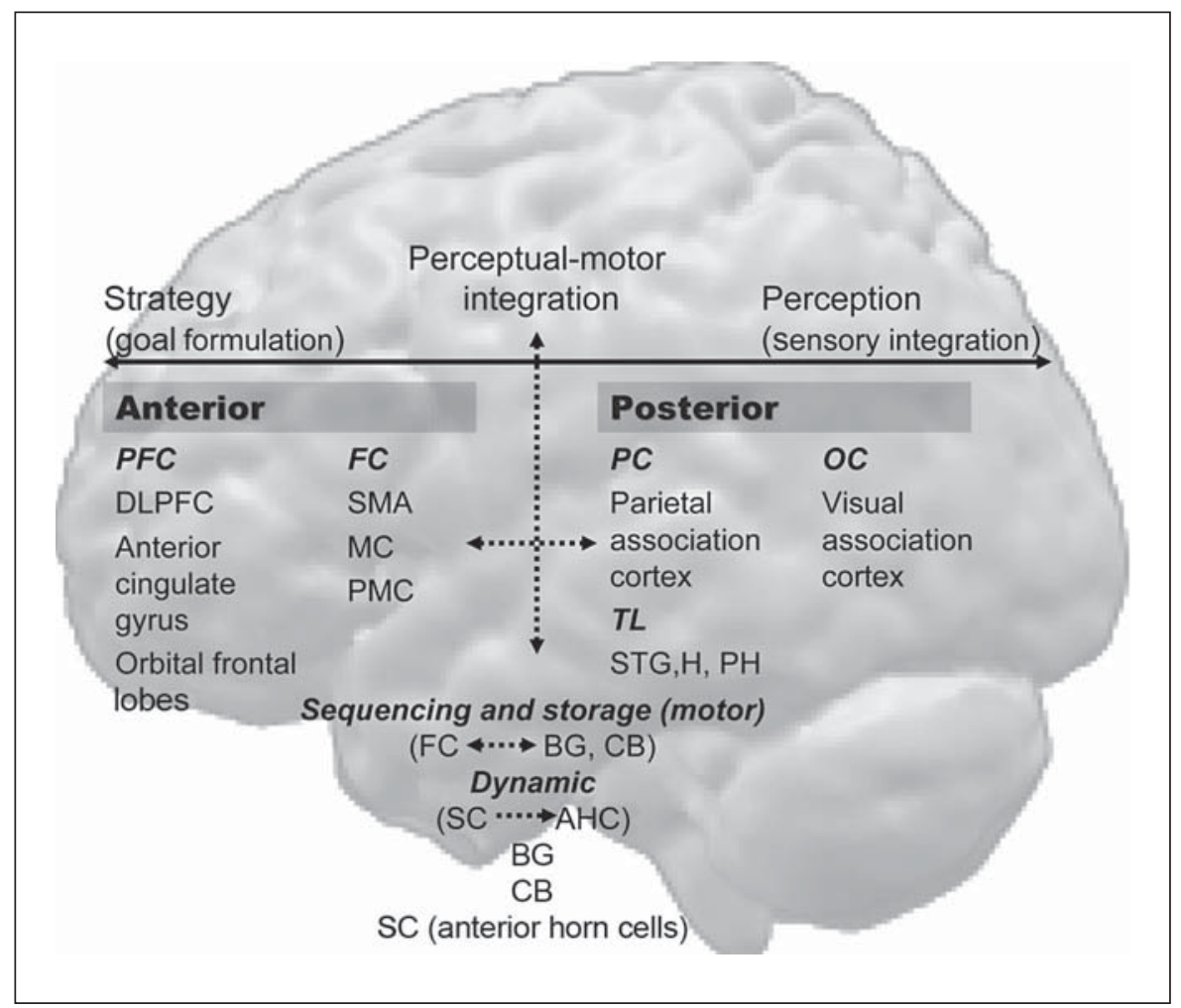

ior [29, 39-43]. These include initiation or intention of action, planning, working memory, and attention. Attention is a dynamic function driven by sensory perception and the need to select a preferred stimulus for a particular action while ignoring the unnecessary and the irrelevant [41-43]. The process of attention is divided into separate functions such as orienting, shifting, response selection, divided attention, and vigilance [41-43].

In the 'supervisory attention system' model of executive function, two basic control mechanisms determine how activities are executed [42, 43]. On the first, lower level, the so-called 'contention scheduling system' includes over-learned and automatic behaviors. The second, higher level, termed the 'supervisory attention system', deals with modulation of the activities of the first level in a flexible or adaptive way, enabling these schemata to be run as new activities or stopping ongoing activity by a selection process that adds either activation or inhibition.

The level of involvement of cognitive function in motor control, particularly executive function, depends on the goal of motor behavior. Significant attention processing of all types and active working memory are absolutely necessary when learning new actions or consciously modifying old programs, such as when attempting to perfect a previously acquired motor skill. Performing routine programs such as walking will require use of attention and executive function, particularly if there is a change in incoming sensory information such as a sudden change of the walking surface (broken concrete) or a sudden decrease in vision or hearing. During senescence, loss of hearing and vision is a common occurrence. Integrity of executive function capacity becomes more important as it is necessary to re-adjust previously learned motor strategies, perceptual motor integration and dynamics of motor programs. Perceptual 'fine tuning' and adjustment become a more active process in the setting of primary sensory impairment [44-46]. Previous programs or visual-spatial 'maps' have to be re-adjusted, requiring more active involvement of polymodal sensory association areas in the brain such as the posterior parietal lobes and the superior temporal gyrus along with the hippocampus $[44,45]$. So in the setting of dementia a higher possibility of falling might occur because of inability to adapt to the common sensory losses of aging.

In $\mathrm{AD}$, executive dysfunction and loss of attention control are now considered pervasive [41, 47-59]. Results from a prospective neuropathologic study of 31 normal 
controls enrolled in a longitudinal aging project at an $\mathrm{AD}$ center demonstrated a progressive decline of executive function in 14 subjects found to have AD at autopsy [41]. Progressive decline in neuropsychological measurements of executive functions along with some memory functions were the only areas of cognition found to correlate with development of $\mathrm{AD}$.

Attention deficits are prominent at all stages of $\mathrm{AD}$ $[41,48,52,53]$. A prospective study of subjects with minimal and mild AD (MMSE scores between 24-30 as 'minimal' and 18-23 as 'mild') suggested that selective attention is the area of attention capacity affected early in the disease, after episodic memory [53]. Psychometric batteries were selected on their ability to differentiate between tests that rely on posterior attention network functions (temporal-parietal, i.e. map search and visual object space perception) and those with predominant prefrontal input (the Stroop test and Wisconsin Card Sort). They demonstrated significant differences on performance between controls versus the mild AD cohort $(p<0.0001)$ and minimal versus mild $(p<0.001)$ on most batteries.

In one current model of working memory [54, 56-59], the 'central executive system' coordinates attention and information flow to and from verbal and spatial shortterm memory slave systems, the 'articulatory loop system' and the 'visuospatial sketchpad', respectively. Although the functioning of the articulatory loop system and visuospatial sketchpad is known to be impaired in moderate to severe $\mathrm{AD}$, it is in the operations of the central executive system that the most profound and earliest dysfunction is thought to occur. According to this explanation, the capacity of the central executive system is limited, and when tasks become more complex, this capacity is exceeded and performance starts to break down [54, 56-59].

When healthy subjects perform two tasks simultaneously and are required to divide attention, they may show a deterioration in performance on task A or B when they are performed together, compared to separate performance. This deterioration in performance is known as a dual-task decrement. A typical dual-task is manual tracking and repeating strings of digits. The term 'dual task' refers to two tasks performed simultaneously (e.g. cognitive task with motor task simultaneously, or two motor tasks in parallel). In a healthy subject, one task may be performed automatically (unconsciously) requiring minimal attention reservoirs, while the person is attentive to the second task. Many studies that used this paradigm have shown that AD patients perform like controls when the two tasks are attempted separately but show a disproportionate decline in performance when the tasks are performed concurrently [57, 58]. It was suggested that the deterioration in performance is a function of whether a single or dual task is being performed and does not depend on task difficulty alone [57, 58]. Also, this deficit has been ascribed to specific dysfunction in $\mathrm{AD}$ of the central executive system [54-59]. Although the vast majority of $\mathrm{AD}$ patients in the moderate stages likely show marked impairment, the stage at which patients show impairment on dual task remains controversial.

\section{Gait Function and Movement Are Abnormal in $A D$}

AD does not usually affect the motor cortex until the later stages of the process; however, a couple of recent neuropathologic studies show a disease burden in the motor cortex equivalent to that of the more commonly recognized targets, such as the entorhinal cortex, hippocampus and the association areas of the frontal and parietal lobes $[60,61]$. These findings are supported by a number of studies using TMS [62-64]. Results from all investigations showed hyperexcitability (lower threshold of stimulation needed to activate) in the motor cortex of AD patients, all mild or moderately impaired (including some with MCI). All participants were not in active treatment with acetylcholinesterase inhibitors during testing. Experimental use of rivastigmine did not change the threshold for excitability (still low compared to age-matched controls) [63]. All authors suggested that motor cortex hyperexcitability may be compensatory and responsible for the lack of apparent clinical dysfunction. The altered physiology may result from abnormal glutamate neurotransmission or loss of cortical inhibition. It was determined that the decreased cholinergic tone found in $\mathrm{AD}$ was not responsible [62-64].

The patterns of walking in persons with $\mathrm{AD}$ are different from those of age-matched, cognitively intact elders [13-15, 65-70]. In a pivotal study reported in 1983, demented patients were shown to have shorter step length, lower gait speed and stepping frequency, greater step-tostep variability and larger sway [14]. Similar findings were described later in a report of gait patterns and balance in 17 patients with AD and 15 controls [67]. There were no extrapyramidal signs or musculoskeletal abnormalities. Compared with the healthy, age-matched adults, AD subjects had slower walking speed, slowness approaching and crossing an obstacle, and other abnormal- 
ities of gait. A comparison of gait patterns in 30 subjects with $\mathrm{AD}$ and vascular dementia $(\mathrm{VaD})$ to age-matched controls showed significantly decreased step length and velocity in those with $\mathrm{AD}$ and $\operatorname{VaD}(\mathrm{p}<0.01$ for both) [69], although the subjects with $\mathrm{VaD}$ performed significantly worse than the AD $(p<0.01, p<0.05)$. Both AD and $\mathrm{VaD}$ subjects exhibited decreased cadence compared to controls; however, it was not significant. The authors concluded that step length was more important than cadence and velocity as a predictor of other variables of walking and that these findings may relate to changes in blood flow in the frontal regions of the cortex.

The concept of gait dysfunction as a persistent feature of $\mathrm{AD}$ was supported by the findings of a cross-sectional study of 55 patients with probable AD at all stages of severity [68]. Accepted criteria for higher cortical gait disorders were used for diagnoses and severity of dementia was determined using the Clinical Dementia Rating Scale (CDR). Subjects were equally distributed amongst each category of severity, mild to severe (CDR 1-3). The prevalence of gait dysfunction increased with severity of dementia; $57 \%$ of the severely demented patients were affected. A large percentage of patients, $40 \%$, compared to $18 \%$ of controls had evidence of a higher cortical gait disturbance $(\mathrm{p}<0.01)$.

The association of gait difficulties with dementia is an evolving concept. The relationship between coexistent subcortical vascular disease, commonly referred to as leukoaraiosis and clinical manifestations observed in AD, both cognitive and non-cognitive is well established [71]. MRI of the brain is now a common tool in the evaluation of memory loss and dementia. Subcortical leukoaraiosis is found in a very high percentage of persons later confirmed to have $\mathrm{AD}$ [72-75] and its is now thought that evolving small vessel disease of the white matter in the cortex is responsible for both clinical and pathologic progression [72,73]. White matter lesions have been divided into two subtypes by some: deep white matter hyperintensities (DWMH) and periventricular hyperintensities (PVH) [75]. DWMH appear to be related to age and vascular risk factors, whereas $\mathrm{PVH}$ are considered to be an epiphenomenon of brain atrophy. A recently published pathological study [72] showed a significant relationship between amyloid angiopathy and arteriosclerosis as it relates to deep white matter disease in 137 patients with autopsy-confirmed AD. It was suggested that shared risk factors, perhaps impaired vascular autoregulation, was a contributing factor. The association between gait dysfunction, leukoaraiosis and vascular dementia is well supported [76] even though the diagnostic criteria are varied and not necessarily interchangeable $[77,78]$. Several recent reports support a strong association between white matter disease and gait impairment in normal elderly individuals $[79,80]$, suggesting that imaging using MRI should be a routine part of an evaluation for gait impairment.

Movement in general is impaired in AD. Individuals with mild cognitive impairment (MCI) and $\mathrm{AD}$ performed worse on all motor tasks than age-matched normal controls [15]. Performance on motor tasks predicted cognitive function as well as tests of memory and language, suggesting that motor and psychomotor assessments may be as sensitive as traditional tests of cognitive function in identifying individuals in the earliest stages of $\mathrm{AD}$. An investigation to determine the difference between bradykinesis in $\mathrm{AD}$ and normal aging demonstrated significantly slowed performance on all measures of motor speed including finger tapping, point-to-point arm movements and gait parameters [70]. These results also showed a positive correlation with cognitive tests of concentration and executive function, suggesting a relationship to frontal lobe disease. Evaluation of limb coordination and equilibrium in 365 individuals found that persons with $\mathrm{MCI}$ or mild $\mathrm{AD}$ had significantly poorer performance on tests of coordination and balance compared with cognitively intact controls $(\mathrm{p}<0.05)$ [81]. A recently published study determined that motor signs of all types were predictive of a poor outcome in $\mathrm{AD}$ [82]. The authors wanted to study the predictive value of motor signs (11 items including tremor, gait, bradykinesis and rigidity) for outcomes of subjects with $\mathrm{AD}$ enrolled in a 13-year longitudinal study. Motor signs predicted a faster and more severe decline in four areas of outcome: cognitive (RR 1.72; 95\% CI 1.24-2.38), functional (RR 1.80, CI 1.33-2.45), institutionalization (1.68; 1.26-2.25) and death $(1.38 ; 1.05-1.82)$. Tremor was associated with increased risk for poor cognitive outcome, bradykinesis functional and postural gait abnormalities increased risk for institutionalization and death.

Apraxia, defined simply, is an inability to perform skilled movements in the face of apparently normal elementary motor and sensory function. The various descriptions and subtypes are less than simple with many nomenclatures applied to the same clinical observations [83]. Loss of praxis in $\mathrm{AD}$, particularly constructional and ideomotor, is a well-documented observation [83, 84]. To the contrary, systematic examination of gait apraxia in $\mathrm{AD}$ is a recent trend [84]. This cross-sectional study examined the relationship between performance on a recently developed test of walking skills: The assessment of 
walking skills (AWS) and dementia severity. The AWS contains features that assess, in detail, both limb and gait apraxia. Cognition was examined using the Milan overall dementia assessment (MODA), a cognitive battery with correlation to the MMSE. The results demonstrated a highly significant relationship between scores on the AWS and dementia severity $(\mathrm{p}<0.0001)$. There was no correlation with patients who self-reported walking difficulties $(p=0.89)$. Stepwise progression also showed that age, dementia severity and limb apraxia scores were independent predictors of walking performance on the AWS $(\mathrm{r}=$ $0.23,0.53$ and 0.50 , respectively). The authors concluded that walking disorders in $\mathrm{AD}$ may result from gait apraxia. The difficulty with the concept of gait apraxia is the variability of clinical descriptions and anatomical associations [83]. Since walking or locomotion involves automatic, repetitive motor function, the concept of gait apraxia remains unsettled.

\section{Gait Unsteadiness and Variability of Stride Timing Are Significant for Fall Risk in AD}

Falling is a markedly common occurrence in AD. The prevalence of falls and associated sequelae markedly exceeds that of cognitively intact, age matched peers [5-9]. A longitudinal study of $\mathrm{AD}$ and normal aging found that falls occurred in $36 \%$ of the subjects with AD compared to $11 \%$ of the age-matched controls [8]. The higher frequency of falls did not correlate with drug use or severity of neurologic deficit. The fallers also had a higher rate of institutionalization, suggesting an association between $\mathrm{AD}$, falls and loss of independence. A prospective study focusing on falls and fractures showed similar results [9]. In a group of $157 \mathrm{AD}$ patients, including 117 followed for a 3-year period 50\% either fell or became unable to walk. At the start of the study, all but 1 patient could walk and $31 \%$ of the cohort reported falling. The fracture rate (69/1,000/year) during follow-up was three times the age- and sex-adjusted rate in the general population.

Sensory loss in multiple modalities is common in senescence and the relationship with falling is complicated. Fall risk is divided into intrinsic (personal) and extrinsic (environmental) factors; personal risk factors included problems with gait and balance, functional impairments, and behavioral (i.e. lack of physical activity, mental illness, drugs) [85]. All of these are directly influenced by primary sensory impairment, for example loss of vision can lead to social isolation, decreased physical activity and depression, all associated with high fall risk. Yet, a recently published longitudinal (10-year) project, designed to identify the causes of deteriorating gait and balance in normal elderly individuals, found weak correlations between age-related decreases in multiple sensory functions and changes in gait and balance [86]. Fifty-nine normal subjects, mean age 78.5 years, were evaluated annually for a period of 10 years. In addition to yearly clinical exams (including neurologic and visual), subjects underwent a detailed gait exam, tests of vestibular function (including VORs), audiograms, lower extremity vibratory function and brain MRIs (evaluated in detail for white matter hyperintensities). Outcome measures were: Tinetti score, gait impairment, number of falls, MMSE and the Purdue pegboard test. Results showed age-related decrements in all sensory modalities and gait function; however, the variable that correlated most significantly with gait impairment (and Tinetti scores) was the volume of white matter hyperintensity $(\mathrm{p}<0.001,0.02$, respectively). The authors concluded that although age-related changes in sensory function affect gait, the impact was not as significant as they expected.

Gait unsteadiness or inconsistency and arrhythmicity of stepping has been found to be one aspect of walking closely associated with risk of falls in the elderly, even more so than gait speed $[10,14,69,87-90]$. This is a finding in multiple studies using a variety of methods for assessing gait, including but not limited to: direct observation of walking, use of visual tags combined with videotape, walking in artificial environments such as gait labs, or ambulatory monitoring using footswitches, accelerometers, gyroscopes and other body-mounted devices [10, $14,69,87-90]$.

Impairment in the ability to maintain a steady gait, with minimal stride-to-stride variations, is closely related to instability and fall risk, and is independent of gait speed and healthy-aging effects.

A recent study of community-dwelling elders, both fallers and non-fallers, evaluated the relationship between multiple temporal parameters of gait and falls using a transducer (footswitch) placed in the shoes [87]. The cohort of elderly 'fallers' had a variation of stride $\sim 10$ times higher than that of young normals and 5 times higher than that of elderly non-fallers $(\mathrm{p}<0.0002)$. Again, the parameter that appeared to coincide with fragility and decline was variation in stride or the pattern and timing of foot placement while walking. This does not appear to be a feature of aging as numerous studies of normal elderly non-fallers compared to young controls have shown that there is no significant difference among the groups in any 
parameters of gait functioning including stride variability [87-89].

A prospective study of the relationship between various aspects of gait and falls in one hundred Japanese patients with $\mathrm{AD}$ reported a significant correlation between the number of falls, stride length variability and severity of dementia [10]. The authors evaluated gait ability in 97 residents of a nursing home, all with $\mathrm{AD}$ CDR 1 (50 patients) and CDR 2 (moderate disease severity, 47 patients). They excluded individuals with known motor disease, arthritis, orthopedic impairments, cataracts, blindness, other diseases of the central nervous system and inability to walk independently. Subjects were examined four times during a 2-year period. The percentage of fallers was significantly higher $(38 \%, \mathrm{p}<$ 0.05 ) in the CDR 2 group compared to the mildly demented group (20\%); variability of stride length, used as a measure of gait instability, was significantly higher in the CDR 2 group $(p<0.01)$ and was the best predictor of falls. The authors concluded that stride length variability alone is an effective index of gait stability and predictor of falls.

\section{Effects of Executive Dysfunction on Gait Instability in AD}

The susceptibility to distraction and other manifestations of impaired attention function observed in the elderly, particularly those with dementia, have been examined in clinical studies using dual task paradigms.

One group examined the phenomena of 'stops walking when talking' [91] in a mixed cohort of community-dwelling older adults, some of whom had dementia, depression and/or were post-stroke. They used dual task paradigms. Subjects who stopped walking while talking had a greater tendency to fall. The same group also found that older adults that require an additional $4.5 \mathrm{~s}$ or more to perform the Timed Up and Go (TuaG) test while performing a manual task have a greater fall risk [92]. According to these two studies, 'stops walking while talking' and dual task differences in the TuaG could be predictors of falls in older adults.

This idea was supported by the findings of a small study in 15 patients with mild AD using a dual task paradigm. Results showed that performing a dual task negatively affects gait in subjects with $\mathrm{AD}$ [14]. Walking speed in the patients slowed while performing a verbal task, i.e., reciting names, suggesting that distraction or impairment of attention may influence walking ability in AD.
It has been argued that while automatic processes are available in the early stages of $\mathrm{AD}$, the increased allocation of attention and increased concentration usually reserved for novel tasks may be needed to perform even familiar activities. When such tasks increase in complexity or are run concurrently, the processing resources needed exceed those available either because of depletion or inefficient deployment. It has been shown that in the performance of cognitive tasks, AD subjects recruit different brain regions compared to controls [93] and these alternative brain regions are also associated with increased task effort [94].

We found that performing a relatively simple dual task (walking and reciting random, single digits) increased variability of stride time, a temporal measure associated with fall risk [12].

We studied 28 patients with AD, equally divided between CDR scale scores of 1-3. Using a footswitch, a computerized insole placed in the shoe, we measured timing of walking under two separate conditions: walking $15.24 \mathrm{~m} \mathrm{(50} \mathrm{ft)} \mathrm{on} \mathrm{a} \mathrm{flat} \mathrm{surface} \mathrm{at} \mathrm{a} \mathrm{self-determined} \mathrm{pace}$ and walking while reciting random single digits (dual-task paradigm).

Variability of stride time increased significantly during the dual task as compared to the simple walking task. When walking while talking, i.e., while performing forward digit span, gait speed decreased by $11.4 \pm 37.1 \%$ or $0.09 \pm 0.18 \mathrm{~m} / \mathrm{s}(\mathrm{p}<0.012)$. The stride-to-stride standard deviation of the stride time increased by $51.7 \pm 72.2 \%$ or $51 \pm 92-170 \pm 99 \mathrm{~ms}(\mathrm{p}<0.002)$. The effect on gait variability was larger than the effect on gait speed $(\mathrm{p}<$ 0.015) (fig. 2).

We also demonstrated a correlation between performance on several measures of executive function (verbal fluency, clock drawing: CLOX I,II), cognitive function in general (MMSE) and variability of gait timing $(p<0.02)$, suggesting that as cognitive function decreases, the ability to maintain a stable gait while performing a simple secondary task decreases in parallel.

\section{Gait Dysfunction as a Marker of Cognitive Decline in AD}

Pathologists have started to document AD pathology in regions of brain primarily involved with motor function [60,61]. Motor abnormalities, particularly extrapyramidal disease such as stooped posture and tremor, are historically associated with AD; however, until recently this was merely a clinical observation, subject to indi- 

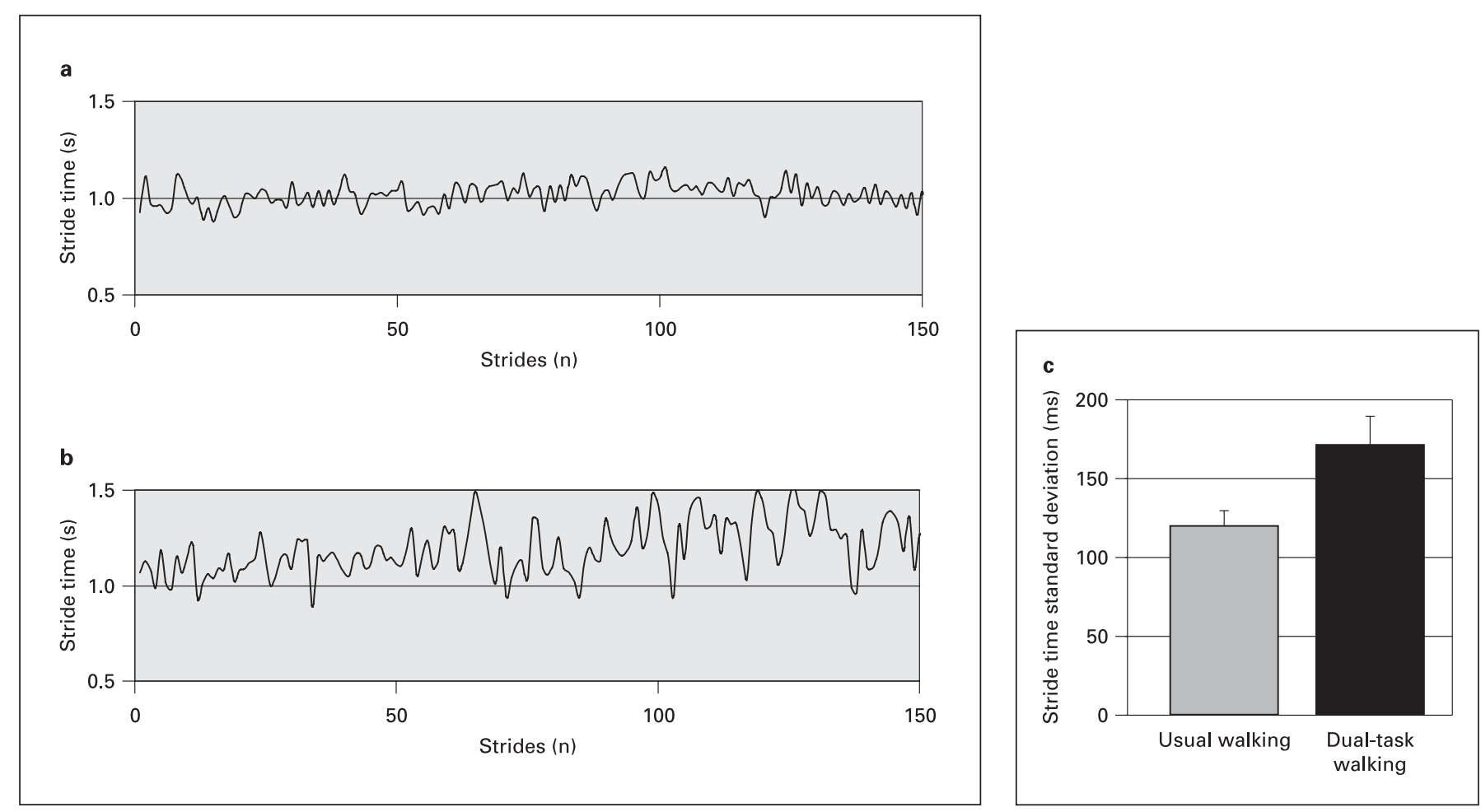

Fig. 2. This is an example of the effect of dual task walking from one subject (a stride time, usual walking; b stride time dual-task walking). For this subject, the stride time variability increased from 59 to $141 \mathrm{~ms}$ (or from 5.6 to $118 \%)$. In general, performance of a dual task markedly decreased $(\mathrm{p}<0.002)$ the ability of patients with AD to regulate the stride-to-stride variations in gait timing (c). The standard deviation of the stride time (the gait cycle duration) increased by $52 \%$ when subjects were asked to walk and talk at the same time. This increase was associated with impaired executive function (see text). Error bars reflect the standard error of the mean. Mean was obtained from all 28 subjects (adapted from Sheridan et al. [12]).

vidual interpretation. The association between motor impairment and severity of dementia is a relatively recent concept.

Reports of gait dysfunction as a predictor of incipient dementia are conflicting. A recently published study documented a strong association between primary gait impairment and risk for future dementia, particularly associated with vascular disease [95]. The relationship between abnormal gait at baseline entry into the cohort and incipient dementia was examined in 422 subjects 75 years of age and older. At enrollment, 85 subjects had neurologic gait abnormalities including frontal, unsteady, hemiparetic, parkinsonian as well as three other types. During follow-up (median duration 6.6 years) 125 or $\sim 30 \%$ developed dementia: $70 \mathrm{AD}, 55$ non-AD (47 vascular, 8 other). Using Cox proportional hazards regression analysis, they determined that any type of gait disorder was an increased risk of dementia (hazard ratio of
$1.96,95 \%$ confidence interval, 1.30-2.96). The risk of AD was 1.07 , but the risk of non-Alzheimer's dementia was 3.51. Abnormal gait in non-AD dementia significantly predicted the development of future dementia: 3.46 hazard ratio (95\% CI, 1.86-6.42). They concluded that the presence of a neurologic gait disorder is significantly predictive of future dementia, particularly non-AD.

In contrast, a pivotal cross-sectional study documented the presence of all types of higher cortical and extrapyramidal gait disorders at all levels of dementia severity in subjects with $\mathrm{AD}$ [68]. The frequency and prevalence increased significantly as the disease progressed and dementia became more severe. They concluded that contrary to our widely accepted criteria for diagnosis, gait dysfunction appears to be a persistent feature of the disease. This is supported by results of a recent examination of the predictive value of gait and motor slowing in relationship to future dementia [96]. Six hundred and thirty 
community-dwelling participants ( 75 years and older) in a longitudinal aging study (The Sydney Older Persons Study) were examined over a 6-year period. The presence of cognitive impairment in association with gait and motor slowing was highly predictive of onset of dementia (OR 5.6; 95\% CI 2.5-12.6) and death (OR 3.3; 95\% CI 1.6-6.9).

\section{Exercise Has Not Been Effective for Preventing Falls in AD}

There have been a fair number of trials attempting to improve various aspects of functioning for patients with $\mathrm{AD}$, especially mobility and prevention of falls [97-100]. The results have been less than encouraging with either equivocal findings or positive results in trials that used exercise mixed with caregiver behavioral interventions.

Results from a randomized, controlled, prospective trial, published in 2003, support the hypothesis that exercise as an intervention to prevent falls in older people with cognitive impairment is not effective [97]. The authors randomized 274 persons between the ages of 71 and 97 years, all with cognitive impairment (MMSE <24) and all with a history of at least one fall that required an emergency evaluation, to either undergo a multi-factorial assessment and intervention protocol: assessment of medical, cardiovascular and mobility along with identification and treatment of fall risk factors, along with a home-based exercise program, or the control protocol: conventional care from the usual primary health providers and specialists. The primary outcome was the number of participants who fell during the year; secondary outcomes included number of falls, emergency room visits, injury rates and mortality. Intention to treat analysis showed no significant differences between intervention and control groups in proportion of patients who fell during the 1-year period: $74 \%(96 / 130)$ and $80 \%(115 / 144)$, relative risk ratio $0.92 \%(95 \% \mathrm{CI} 0.81-1.05)$. Again note the high percentage of fallers in both groups. There also were no significant differences between secondary outcome measures. The authors concluded that use of multifactorial interventions, including exercise, are less effective in older adults with cognitive impairment and that resources would be more judiciously used to improve physical functioning including mobility, in cognitively intact elders, a population that has been shown to have significant benefits from exercise and other fall prevention interventions. It is important to note that a study in nondemented older adults using an identical multi-facto- rial, intervention was effective at reducing falls [101, 102].

This has been the predominant outcome of most trials using exercise as prevention of falls, and, currently all agree that more research is absolutely necessary to address, in detail, the issue of falling and gait instability in dementia and AD. Based on the discussion above, we suggest that improving gait to reduce falls in $\mathrm{AD}$ must include interventions targeting improvement of cognitive functions, especially higher-level and executive function.

\section{Conclusions}

Walking appears to be an automatic process until it is necessary to deviate from the learned program. As most human movement is associated with goals, most locomotion involves intention and therefore cognitive input of various degrees.

Since this is the case, cognitive impairment will have a negative effect on all aspects of gait performance.

In brain diseases that cause dementia, progressive decline in cognition may cause concomitant disorganization of the network that controls locomotion, leading to impaired gait timing and postural control.

In $\mathrm{AD}$ in particular this might represent a disintegration of higher cortical sensory function, particularly involving perceptual-motor integration. Previously learned schemata for routine motor functions such as walking 'break down' due to disintegration of the various components such as visuospatial integration and other functions of higher cortical perception, an unconscious component in the network of motor control. This is supported by the presence of both constructional and ideomotor apraxia in AD patients at all stages of the disease. Alternatively, as a result of an attempt to compensate for impaired higher cortical sensory integration and competition for attentional resources, the control of timing in the cerebellum executes programs with variable output, leading to variability of stepping during stride with subsequent gait unsteadiness and ultimately falls.

The temporal disorganization of gait timing may also reflect the pervasive loss of executive function, particularly attention capacity and working memory. Attention, particularly response selection, and the ability to divide attention are impaired early in AD. Working memory is one component of executive control found to be necessary for sequential ordering of movement. Given the essential role of the hippocampus and parahippocampal regions in 
managing novel perception, the pervasive impairment of working memory long observed clinically may actually result from damage to the hippocampus. All actions can be considered 'novel' or nonroutine if the environment in which they occur presents 'novel' challenges such as water on the floor or changes in lighting. For the adult with AD this may lead to instability of an automatic motor program such as walking because of faulty perceptualmotor integration.

Exercise as an intervention for falls and apparent motor dysfunction is not effective in AD. Clinical trials should focus on a pharmaceutical therapy that maximizes both cognitive and motor function. The positive effects of methylphenidate on attention are well known from widespread use in attention deficit disorder and attention deficit/hyperactivity disorder. Methylphenidate, a dopamine reuptake blocker and an inhibitor of catecholamine reuptake, is a widely available, inexpensive treatment option in use as empiric treatment of apathy and depression in the elderly including those with $\mathrm{AD}$ [103-105]. Results of a pilot study $(n=5)$ to determine the effects of methylphenidate as an adjunct to $L$-DOPA in Parkinson's disease (PD) demonstrated an increase in the motor effects of $L$-DOPA with the addition of methylphenidate. There were minimal effects on cognition and affect (with the exception of reaction time) [106]. Chronic administration of nicotine via transdermal patch has been found to have positive effects on cognition in $\mathrm{AD}$ and MCI, particularly in the subset of attention [107]. Results from a 4-week double-blind, placebo-controlled, cross-over study reported in 1999 showed a significant improvement in performance on continuous performance tests and components of reaction time tests in subjects with AD [107]. Similar results were also demonstrated in a later study of the same design with subjects diagnosed as age-associated memory impairment (or MCI) [107]. Nicotine improved the results of the Clinical Global Impression Score along with performance on at- tentional tests of the continuous performance tests. Abnormalities of glutamate transmission and N-methyl- $D$ aspartate (NMDA) receptor activity are implicated in a variety of neuropsychiatric disorders including $\mathrm{AD}[108$, 109]. Competitive antagonism of NMDA receptors results in disruption of long-term potentiation and other memory functions along with adverse effects such as hallucinations; however, noncompetitive blockade using agents such as ketamine and memantine improves performance in animal models (aged rats and transgenic mouse models of $\mathrm{AD}$ ) and in humans with dementia and AD [108]. A double-blind, placebo-controlled investigation, reported in 2005, showed selective impairments of cognition in normal subjects [109]. Ketamine was administered to 10 healthy, young volunteers during two sessions. Learning and memory was examined using a virtual Morris water task along with the Hopkins Verbal learning Test; attention was assessed using the Stroop; working memory (digit span) and verbal fluency (FAS). Ketamine impaired learning of spatial and verbal memory and spatial working memory; attention, verbal fluency and verbal working memory were not affected. Neuropsychiatric symptoms such as visual alterations, auditory alterations, dreamlike states and altered time perception occurred in all participants.

In $\mathrm{AD}$ an approach that specifically targets executive dysfunction, particularly attention, may help to reduce the high rate of falls and decrease the associated morbidity in this fast-growing older adult population.

\section{Acknowledgments}

This work was supported in part by NIH grants AG-14100, RR13622, HD-39838 and AG-08812 and a fellowship from the Clinical Investigator Training Program: Beth Israel Deaconess Medical Center-Harvard/MIT Health Sciences and Technology, in collaboration with Pfizer, Inc.

\section{References}

1 Jellinger KA: The neuropathological diagnosis of Alzheimer's disease. J Neural Transm Suppl 1998;53:97-118.

2 Braak H, Braak E: Diagnostic criteria for neuropathologic assessment of Alzheimer's disease. Neurobiol Aging 1997;8:S85-S88.

3 Dietz V: Neurophysiology of gait disorders: present and future applications. Electroencephal Clin Neurophysiol 1997;103:333-355.

Higher-Level Cognitive Function in Gait
4 Shik ML, Orlovsky GN: Neurophysiology of locomotor automatism. Physiol Rev 1976;36: $465-501$

$\checkmark 5$ Imamura T, et al: Fall-related injuries in dementia with Lewy bodies (DLB) and Alzheimer's disease. Eur J Neurol 2000;7:77-79.

-6 Morris JC, Rubin EH, Morris EJ, Mandel SA: Senile dementia of the Alzheimer's type: an important risk factor for serious falls. J Gerontol 1987;42:412-417.

\footnotetext{
7 Koutsavlis AT, Wolfson C: Elements of mobility as predictors of survival in elderly patients with dementia: findings from the Canadian Study of Health and Aging. Chronic Dis Can 2000;21:93-103.

8 Weller I, Schatzker J: Hip fractures and Alzheimer's disease in elderly institutionalized Canadians. Ann Epidemiol 2004;14:319324.
} 
-9 Buchner DM, Larson EB: Falls and fractures in patients with Alzheimer-type dementia. JAMA 1987;257:1492-1495.

10 Nakamura T, Meguro K, Sasaki H: Relationship between falls and stride length variability in senile dementia of the Alzheimer type. Gerontology 1996;42:108-113.

$\checkmark 11$ Camicioli R, Howieson D, Lehman S, Kaye J: Talking while walking: the effect of a dual task in aging and Alzheimer's disease. Neurology 1997;48:955-958.

12 Sheridan P, Solomont J, Kowall N, Hausdorff JM: Influence of executive function on locomotor function: divided attention increases gait variability in Alzheimer's disease. J Am Geriatr Soc 2003;51:1633-1637.

13 O'Keeffe ST, et al: Gait disturbance in Alzheimer's disease: a clinical study. Age Ageing 1996;25:313-316.

14 Visser H: Gait and balance in senile dementia of Alzheimer's type. Age Ageing 1983;12:296301 .

15 Kluger A, et al: Patterns of motor impairment in normal aging, mild cognitive decline, and early Alzheimer's disease. J Gerontol B Psychol Sci Soc Sci 1997;52:28-39.

16 Beloozerova IN, Sirota MG: Cortically controlled gait adjustments in the cat. Ann NY Acad Sci 1998;16:860:550-553.

17 Willingham D: A neuropsychological theory of motor skill learning. Psychol Rev 1998;105: 558-584.

-18 Christensen LO, Johannsen P, Sinkjaer T, et al: Cerebral activation during bicycle movements in man. Exp Brain Res 2000;135:6672.

-19 Fukuyama H, Ouchi Y, Matsuzaki S, Nagahama Y, et al: Brain functional activity during gait in normal subjects: a SPECT study. Neurosci Lett 1997;228:183-186.

20 Malouin F, Richards CL, Jackson PL, Dumas F, Doyon J: Brain activations during motor imagery of locomotor related tasks: a PET study. Hum Brain Mapp 2003;19:47-62.

-21 Hikosaka O, Nakamura K, Sakai K, Nakahara $\mathrm{H}$ : Central mechanisms of motor skill learning. Curr Opin Neurobiol 2002;12:217-222.

-22 Jahanshahi M, et al: Self-initiated versus externally triggered movements. I. An investigation using measurement of regional cerebral blood flow with PET and movement-related potentials in normal and Parkinson's disease subjects. Brain 1995;118:913-933.

23 Jueptner M, Frith CD, Brooks DJ, et al: Anatomy of motor learning. II. Subcortical structures and learning by trial and error. J Neurophysiol 1997; 77:1325-1337.

24 Passingham RE: Attention to action. Philos Trans R Soc Lond B Biol Sci 1996;351:14731479.

25 Jueptner M, et al: Anatomy of motor learning. I. Frontal cortex and attention to action. J Neurophysiol 1997;77:113-1324.

26 Johansen-Berg H, Matthews PM: Attention to movement modulates activity in sensori-motor areas, including primary motor cortex. Exp Brain Res 2002;142:13-24.
27 Jahanshahi M, Frith CD: Willed action and its impairments. Cognitive Neuropsychol 1998; 15:483-533.

28 de Jong BM, van Zomeren AH, Willemsen ATM, Paans AMJ: Brain activity related to serial cognitive performance resembles circuitry of higher order motor control. Exp Brain Res 1996;109:136-140.

29 Badgaiyan RD: Executive control, willed actions, and non-conscious processing. Hum Brain Mapp 2000;9:38-41.

30 Gopher D: Attention control: explorations of the work of an executive controller. Brain Res Cogn Brain Res 1996;5:23-38.

31 D'Esposito M, Ballard D, Zarahn E, Aguirre GK: The role of prefrontal cortex in sensory memory and motor preparation: an event-related fMRI study. Neuroimage 2000;11:400408.

32 Rowe J, Friston K, Frackowiak R, Passingham R: Neuroimage 2002;17:988-998.

33 Criado JM, de la Fuente A, Heredia M, Riolobos AS, Yajeya J: Electrophysiological study of prefrontal neurones of cats during a motor task. Pflügers Arch 1997;434:91-96.

-34 Knight RT: Contribution of human hippocampal region to novelty detection. Nature 1996 383:256-259.

35 Tesche CD, Karhu J: Theta oscillations index human hippocampal activation during a working memory task. Proc Natl Acad Sci USA 2000;97:919-924.

36 Sinnamon HM: Hippocampal theta activity related to elicitation and inhibition of approach locomotion. Behav Brain Res 2005; 160:236249.

37 Song EY, Kim YB, Kim YH, Jung MW: Role of active movement in place specific firing of hippocampal neurons. Hippocampus 2005;15: 8-17.

38 Zarahn E, Rakitin B, Abela D, et al: Positive evidence against human hippocampal involvement in working memory maintenance of familiar stimuli. Cereb Cortex 2005;15:303316.

39 Adams RL, Parsons OA: Neuropsychology for Clinical Practice: Etiology, Assessment, and Treatment of Common Neurologic Disorders. Washington, American Psychological Association, 2003.

40 Fuster JM: Synopsis of function and dysfunction of the frontal lobe. Acta Psychiatr Scand 1999;395(suppl):51-57.

41 Perry RJ, Hodges JR: Attention and executive deficits in Alzheimer's disease. A critical review. Brain 1999; 122:383-404.

42 Shallice T, Burgess P: The domain of supervisory processes and temporal organization of behaviour. Philos Trans R Soc Lond B Biol Sci 1996;351:1405-1411.

43 Shallice T: From neuropsychology to mental structure. Cambridge, Cambridge University Press, 1988.

44 Neville H, Bavelier D: Human brain plasticity: evidence from sensory deprivation and altered language experience. Prog Brain Res 2002;138: $177-188$.
45 Bavelier D, Neville HJ: Cross-modal plasticity: where and how? Nat Rev Neurosci 2002;3: 443-452.

46 Theoret H, Merabet L, Pascual-Leone A: Behavioral and neuroplastic changes in the blind: evidence for functionally relevant cross-modal interactions. J Physiol (Paris) 2004;98:221233.

47 Hulette CM, et al: Neuropathological and neuropsychological changes in 'normal' aging: evidence for preclinical Alzheimer disease in cognitively normal individuals. J. Neuropathol Exp Neurol 1998;57:1168-1174.

48 Foldi NS, Lobosco JJ, Schaefer LA: The effect of attentional dysfunction in Alzheimer's disease: theoretical and practical implications. Semin Speech Lang 2002;23:139-150.

49 Lafleche G, Albert MS: Executive function deficit in mild Alzheimer's disease. Neuropsychology 1995;9:313-320.

50 Binetti G, et al: Executive dysfunction in early Alzheimer's disease. J Neurol Neurosurg Psychiatry 1996;60:91-93.

$\checkmark 51$ Albert MS: Cognitive and neurobiologic markers of early Alzheimer disease. Proc Natl Acad Sci USA 1996;93:13547-13551.

52 Baddeley AD, Baddeley HA, Bucks RS, Wilcock GK: Attentional control in Alzheimer's disease. Brain 2001;124:1492-1508.

53 Perry RJ, Watson P, Hodges JR: The nature and staging of attention dysfunction in early (minimal and mild) Alzheimer's disease: relationship to episodic and semantic memory impairment. Neuropsychologia 2000;38:252271.

54 Baddeley A, Logie R, Bressi S, et al: Dementia and working memory. Q J Exp Psychol A 1986; 38:603-618.

55 Baddeley A, Della Sala S, Spinnler H: The twocomponent hypothesis of memory deficit in Alzheimer's disease. J Clin Exp Neuropsychol 1991;13:372-380

56 Baddeley AD: Working memory. Oxford, Oxford University Press, 1986.

$\checkmark 57$ Grober E, Sliwinski MJ: Dual-task performance in demented and nondemented elderly. J Clin Exp Neuropsychol 1991;13:667-676.

$>58$ Della Sala S, Baddeley A, Papagno C, Spinnler $\mathrm{H}$ : Dual-task paradigm: a means to examine the central executive. Ann NY Acad Sci 1995; 769:161-171.

59 Baddeley AD, Bressi S, Della Sala S, Logie R, Spinnler H: The decline of working memory in Alzheimer's disease. A longitudinal study. Brain 1991;114:2521-2542.

60 Suva D, Favre I, Kraftsik R, et al: Primary motor cortex involvement in Alzheimer disease. $\mathrm{J}$ Neuropathol Exp Neurol 1999;58:11251134.

61 Golaz J, Bouras C, Hof PR: Motor cortex involvement in presenile dementia: report of a case. J Geriatr Psychiatry Neurol 1992;5:8592.

62 Ferreri F, Pauri F, Pasqualetti P, et al: Motor cortex excitability in Alzheimer's disease: a transcranial magnetic stimulation study. Ann Neurol 2003;53:102-108. 
63 Di Lazzaro V, Oliviero A, Pilato F, et al: Motor cortex hyperexcitability to transcranial magnetic stimulation in Alzheimer's disease. J Neurol Neurosurg Psychiatry 2004;75:555559.

64 Alagona G, Ferri R, Pennisi G, et al: Motor cortex excitability in Alzheimer's disease and in subcortical ischemic vascular dementia. Neurosci Lett 2004;362:95-98.

65 Rossor MN, et al: Progressive frontal gait disturbance with atypical Alzheimer's disease and corticobasal degeneration. J Neurol Neurosurg Psychiatry 1999;67:345-352.

-66 Alexander NB: Postural control in older adults. J Am Geriatr Soc 1994;42:93-108.

-67 Alexander NB, et al: Maintenance of balance, gait patterns, and obstacle clearance in Alzheimer's disease. Neurology 1995;45:908914.

68 Nutt JG, Marsden CD, Thompson PD: Human walking and higher-level gait disorders, particularly in the elderly. Neurology 1993;43: 268-279.

-69 Tanaka A, Okuzumi H, Kobayashi I: Gait disturbance of patients with vascular and Alzheimer-type dementias. Percept Mot Skills 1995;80:735-738.

-70 Ott BR, Ellias SA, Lannon MC: Quantitative assessment of movement in Alzheimer's disease. J Geriatr Psychiatry Neurol 1995;8:7175.

71 Korczyn AD: The underdiagnosis of the vascular contribution to dementia. J Neurol Sci 2005;229-230:3-6.

-72 Tian J, Shi J, Bailey K, Mann DM: Relationships between arteriosclerosis,cerebral amyloid angiopathy and myelin loss from cerebral cortical white matter in Alzheimer's disease. Neuropathol Appl Neurobiol 2004;30:45-56.

-73 Capizzano AA, Acion L, Bekinschtein T, et al: White matter hyperintensities are significantly associated with cortical atrophy in Alzheimer's disease. J Neurol Neurosurg Psychiatry 2004; 75:822-827.

74 Sitoh YY, Sitoh YY, Sahadevan S: Clinical significance of cerebral white matter lesions in older Asians with suspected dementia. Age Ageing 2004;33:65-71.

75 Kono I, Mori S, Nakajima K, et al: Do white matter changes have clinical significance in Alzheimer's disease? Gerontology 2004;50: 242-246.

76 van Gijn J: Leukoaraiosis and vascular dementia. Neurology 1998;51(suppl 3):S3-S8.

-77 Pohjasvaara T, Mantyla R, Ylikoski R, et al: Comparison of different clinical criteria (DSMIII, ADDTC, ICD-10, NINDS-ARIEN, DSMIV) for the diagnosis of vascular dementia. Stroke 2000;31:2952-2957.

78 Kuller LH, Lopez OL, Jagust WJ, et al: Determinants of vascular dementia in the Cardiovascular Health Cognition Study. Neurology 2005;64:1548-1552.
79 Wolfson L, Wei X, Hall CB, et al: Accrual of MRI white matter abnormalities in elderly with normal and impaired mobility. J Neurol Sci 2005;232:23-27.

80 Wolfson L: Gait and balance dysfunction: a model of the interaction of age and disease. Neuroscientist 2001;7:178-183.

81 Franssen EH, Souren LE, Torossian CL, Reisberg B: Equilibrium and limb coordination in mild cognitive impairment and mild Alzheimer's disease. J Am Geriatr Soc 1999; 47:463469.

82 Scarmeas N, Albert M, Brandt J, et al: Motor signs predict poor outcomes in Alzheimer's disease. Neurology 2005;64:1696-1703.

83 Zadikoff C, Lang AE: Apraxia in movement disorders: a review. Brain 2005 Jun 1; [Epub ahead of print].

84 Della Sala S, Spinnler H, Venneri A: Walking difficulties in patients with Alzheimer's disease might originate from gait apraxia. J Neurol Neurosurg Psychiatry 2004;75:196-201.

-85 Stevens JA, Olson S: Reducing falls and resulting hip fractures among older women. MMWR Morb Mortal Wkly Rep 2000;49:1-12.

-86 Baloh RW, Ying SH, Jacobson KM: A longitudinal study of gait and balance dysfunction in normal older people. Arch Neurol 2003;60: 835-839.

87 Hausdorff JM, Rios D, Edelberg HK: Gait variability and fall risk in community-living older adults: a 1-year prospective study. Arch Phys Med Rehabil 2001;82:1050-1056.

88 Hausdorff JM, Edelberg HK, Mitchell SL, et al: Increased gait unsteadiness in communitydwelling elderly fallers. Arch Phys Med Rehabil 1997; 78:278-283.

89 Maki BE: Gait changes in older adults: predictors of falls or indicators of fear. J Am Geriatr Soc 1997;45:313-320.

90 Azizah MG, Lajoie Y, Teasdale N: Step length variability at gait initiation in elderly fallers and non-fallers, and young adults. Gerontology 2003;49:21-26.

\$1 Lundin-Olsson L, Nyberg L, Gustafson Y: 'Stops walking when talking' as a predictor of falls in elderly people. Lancet 1997;349:617.

$\$ 92$ Lundin-Olsson L, Nyberg L, Gustafson Y: Attention, frailty, and falls: the effect of a manual task on basic mobility. J Am Geriatr Soc 1998;46:758-761.

93 Becker JT, et al: Compensatory reallocation of brain resources supporting verbal episodic memory in Alzheimer's disease. Neurology 1996;46:692-700.

94 Furey ML, et al: Cholinergic stimulation alters performance and task-specific regional cerebral blood flow during working memory. Proc Natl Acad Sci USA 1997;94:6512-6516.

95 Verghese J, et al: Abnormality of gait as a predictor of non-Alzheimer's dementia. N Engl $\mathrm{J}$ Med 2002;347:1761-1768.
96 Waite LM, Grayson DA, Piguet O, et al: Gait slowing as a predictor of incident dementia: 6year longitudinal data from the Sydney Older Persons Study. J Neurol Sci 2005;229-230: 89-93.

97 Shaw FE, Bond J, Richardson DA, et al: Multifactorial intervention after a fall in older people with cognitive impairment and dementia presenting to the accident and emergency department: a randomised controlled trial. BMJ 2003;326:1-6.

-98 Ray WA, Taylor JA, Meador KG, et al: A randomized trial of a consultation service to reduce falls in nursing homes. JAMA 1997;278: 557-562.

-99 Mulrow CD, Gerety MB, Kanten D, et al: A randomized trial of physical rehabilitation for very frail nursing home residents. JAMA 1994;271:519-524.

100 Teri L, Gibbons LE, McCurry SM, et al: Exercise plus behavioral management in patients with Alzheimer disease: a randomized controlled trial. JAMA 2003;290:20152022.

101 Robertson MC, Campbell AJ, Gardner MM, et al: Preventing injuries in older people by preventing falls: a meta-analysis of individual-level data. J Am Geriatr Soc 2002;50:905911.

102 Barnett A, Smith B, Lord SR, et al: Community-based group exercise improves balance and reduces falls in at-risk older people: a randomised controlled trial. Age Ageing 2003; 32:407-414

103 Wallace AE, Koford LL, West AN: Doubleblind, placebo controlled trial of methylphenidate in older, depressed, medically ill patients. Am J Psychiatry 1995;152:929-931.

104 Kittur S, Hauser P: Improvement of sleep and behavior by methylphenidate in Alzheimer's disease. Am J Psychiatry 1999;156:11161117.

105 Leonard BE, McCartan D, White J, King DJ: Methylphenidate: a review of its neuropharmacological, neuropsychological and adverse clinical effects. Hum Psychopharmacol 2004; 19:151-180.

106 Camicioli R, Lea E, Nutt JG, Sexton G, Oken BS: Methylphenidate increases the motor effects of $L$-Dopa in Parkinson's disease: a pilot study. Clin Neuropharmacol 2001;24:208213.

107 White HK, Levin ED: Chronic four-week nicotine skin patch treatment effects on cognitive performance in Alzheimer's disease. Psychopharmacology 1999;84:158-165.

108 Minkeviciene R, Bannerjee P, Tanila H: Memantine improves spatial learning in a transgenic mouse model of Alzheimer's disease. J Pharmacol Exp Ther 2004;311:677-682.

109 Rowland LM, Astur RS, Jung RE, et al: Selective cognitive impairments associated with NMDA receptor blockade in humans. Neuropsychopharmacology 2005;30:633-639. 